\title{
Исследование параметров двумерного электронного газа в квантовых ямах InGaN/GaN методом терагерцового плазмонного резонанса
}

\author{
(C) Е.Р. Бурмистров, Л.П. Авакянц \\ Московский государственный университет им. М.В. Ломоносова (физический фракультет), \\ 119991 Москва, Россия \\ E-mail: eugeni.conovaloff@yandex.ru
}

Поступила в Редакцию 26 мая 2021 г.

В окончательной редакции 17 июня 2021 г.

Принята к публикации 22 июня 2021 г.

Предложен новый подход к определению параметров двумерного электронного газа в квантовых ямах $\mathrm{InGaN} / \mathrm{GaN}$. В основе лежит метод терагерцовой спектроскопии с временны́м разрешением, в рамках которого проводилась регистрация терагерцовых частот двумерных плазмонных резонансов, возбуждаемых в исследуемых образцах гетероструктур InGaN/AlGaN/GaN фемтосекундными лазерными импульсами на длине волны 797 нм. Показано осциллирующее поведение мощности выходного терагерцового излучения с минимумами в диапазоне частот 1-5 ТГц, что связано с возбуждением плазмонных колебаний в двумерном электронном газе, локализованном в квантовой яме $\mathrm{InGaN} / \mathrm{GaN}$. В процессе обработки терагерцовых спектров обнаружен эффект перенормировки эффективной массы двумерного электронного газа, а также модуляции фазы вблизи частот плазмонных резонансов с увеличением температуры образца от 90 до $170 \mathrm{~K}$. Предложенный метод является бесконтактным и может быть использован в широком диапазоне температур.

Ключевые слова: гетероструктура, плазмонный резонанс, квантовая яма, время релаксации, спектроскопия.

DOI: 10.21883/FTP.2021.11.51561.9685

\section{1. Введение}

Гетероструктуры InGaN/AlGaN/GaN находят широкое применение в приложениях физики полупроводников и терагерцовой оптоэлектроники. Большая $(0.42$ эВ) величина разрыва зоны проводимости в сочетании с эффектом пьезолегирования на гетерогранице позволили применять композитные структуры InGaN/AlGaN/GaN в качестве чувствительных детекторов терагерцового (ТГц) диапазона излучения [1]. Указанные особенности также привели к возможности резонансного управления ТГц-излучением за счет возбуждения плазмонных волн в таких структурах. Высокая $\left(\sim 10^{8} \mathrm{~cm} / \mathrm{c}\right)$ скорость дрейфа насыщения, широкая ( 3.34 эВ) запрещенная зона и большое ( $\sim 4.5 \mathrm{MB} / \mathrm{cm})$ значение напряженности поля пробоя [2], достигаемые в гетероструктурах InGaN/AlGaN/GaN при комнатных температурах, позволили применять их в качестве активной среды в мощных квантово-каскадных лазерах и УФ-светодиодах [3]. В эпитаксиальных композитах InGaN/AlGaN/GaN, выращенных на высокочастотных транзисторах (см., например, [4]), подвижность двумерного электронного газа (2ДЭГ) при комнатной температуре достигла значения $3200 \mathrm{~cm}^{2} / \mathrm{B} \cdot \mathrm{c}$.

Эффективность работы УФ-светодиодов с квантовыми ямами (КЯ) InGaN/GaN зависит от динамических параметров 2ДЭГ, определение которых является актуальной исследовательской задачей. В качестве теоретических методов применяют аппарат квантовой механики, основанный на расчетах матричных элементов потенциалов взаимодействий в модели квантовой ямы (КЯ) с конечной высотой энергетических барьеров.

Однако сами по себе подобные вычисления довольно сложны и громоздки и не исключают возможность возникновения погрешности, искажающей достоверность результатов. Среди экспериментальных методов успешно зарекомендовали себя в исследовании электрических свойств 2ДЭГ в квантовых ямах $\mathrm{InGaN} / \mathrm{GaN}$ эксперименты по циклотронному резонансу и измерению эффекта Шубникова-де Гааза [5], которые, однако, проводятся преимущественно при температурах жидкого гелия. В области более высоких температур возможно использовать оптические методы, основанные на спектроскопии в дальней ИК области. Это объясняется усилением интенсивности междолинного фононного рассеяния 2ДЭГ в активном слое $\mathrm{InGaN} / \mathrm{GaN}$ в области высоких температур, а также влиянием случайных флуктуаций сплава, которые приводят к появлению дополнительных центров рассеяния. Несмотря на ряд существующих методов исследования электрических свойств 2ДЭГ в КЯ InGaN/GaN, терагерцовая спектроскопия с временны́ разрешением (THz-TDS) [6] является одним из перспективных способов изучения особенностей конденсированных сред. Эффективность метода THz-TDS заключается в том, что многие характерные особенности, присущие изучаемым структурам, наблюдаются только в терагерцовом диапазоне излучения. Такими характерными особенностями в случае полупроводниковых гетероструктур InGaN/AlGaN/GaN могут быть подвижность и перестройка 2ДЭГ с конечным временем 
в ответ на внешнее ультракороткое возмущение, уровни энергетических подзон и степень их непараболичности. Генерация терагерцового излучения наблюдается при взаимодействии образца с пучком стробирующих фемтосекундных лазерных импульсов с энергией, превышающей ширину запрещенной зоны полупроводникового соединения.

Информацию о параметрах образца несут в себе амплитуда и фаза составляющих частот ТГц-излучения, которые могут быть обнаружены и изучены на основании быстрого фурье-преобразования ТГц-спектров.

В настоящей работе впервые применен метод ТГцспектроскопии с временны́м разрешением для численной оценки параметров 2ДЭГ в КЯ InGaN/GaN. Сущность метода заключается в регистрации частот двумерных (2Д) плазмонных резонансов [7], возбуждаемых в системе 2ДЭГ под действием стробирующих фемтосекундных лазерных импульсов. Далее будет показано, что осциллирующее поведение мощности выходного ТГцизлучения наблюдается в диапазоне частот от 1 до 5 ТГц, что связано с возбуждением в 2ДЭГ коллективных плазмонных волн.

\section{2. Экспериментальные образцы}

Объектом исследования является типичная для светодиодных гетероструктур с квантовыми ямами InGaN/GaN в $p-n$-переходе $\mathrm{GaN}$-слоевая структура. Исследовались готовые образцы промышленных гетероструктур InGaN/AlGaN/GaN, изученные ранее в работе [8] методами модуляционной спектроскопии. Возбуждение плазмонных волн и регистрация их резонансных частот проводились для двух образцов производителей ЗАО „Светлана - оптоэлектроника“ (№ CO1) и ЗАО НПЦ „Полюс“ (№ П1) [8]. Типичная слоевая структура образца представлена на рис. 1.

Из рис. 1 видно, что для гетероструктур InGaN/AlGaN/GaN первыми являются низкотемпературный и буферный слои нелегированного $i$-GaN c толщинами 20 и 0.7 нм соответственно, выращенные

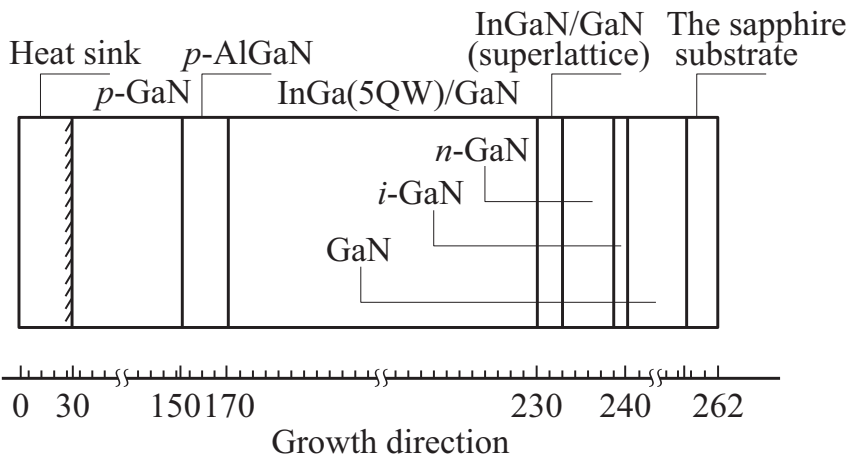

Рис. 1. Слоевая структура полупроводника InGaN/AlGaN/GaN. Показано направление послойного роста c обозначением напыляемых слоев.
Таблица 1. Параметры образцов гетероструктур $\mathrm{In}_{x} \mathrm{Ga}_{1-x} \mathrm{~N} / \mathrm{GaN}$

\begin{tabular}{l|c|c|c|c|c|c}
\hline $\begin{array}{c}\text { Серия } \\
\text { образцов }\end{array}$ & $N$ & $\begin{array}{c}\text { КЯ/КБ, } \\
\text { нм }\end{array}$ & AlGaN & $\begin{array}{c}S, \\
\text { мм² }^{2}\end{array}$ & $x$ & $\begin{array}{c}E_{g}, \\
\text { эВ }\end{array}$ \\
\hline № CO1 & 5 & $\begin{array}{c}3 / 12 \\
\text { № П1 }\end{array}$ & $\begin{array}{c}\text { Есть } \\
\text { Есть }\end{array}$ & 0.4 & 0.24 & 2.6 \\
& 5 & 0.22 & 2.6
\end{tabular}

Таблица 2. Значения энергии квантово-размерного энергетического уровня, реализуемого в активном слое $\mathrm{InGaN} / \mathrm{GaN}$

\begin{tabular}{l|c|c|c|c|c|c}
\hline \multicolumn{2}{c|}{ Серия образцов } & \multicolumn{5}{|c}{ № КЯ } \\
\cline { 3 - 7 } \multicolumn{2}{c|}{} & 1 & 2 & 3 & 4 & 5 \\
\hline № CO1 & $E_{1}$, эВ & 2.74 & 2.88 & 2.88 & 2.88 & 2.74 \\
№ П1 & $E_{1}$, эВ & 2.81 & 2.92 & 2.92 & 2.92 & 2.81
\end{tabular}

методом газофазной эпитаксии (ГФЭ) на сапфировой подложке (толщина 10 мкм) в направлении [0001]. Далее, следует слой $n$ - $\mathrm{GaN}$, легированный кремнием с концентрацией доноров $N_{D}=5 \cdot 10^{18} \mathrm{~cm}^{-3}$ толщиной 3.6 мкм для образца $3 \mathrm{AO}$ „Светлана - оптоэлектроника“ и 4.5 мкм для образца ЗАО НПЦ „Полюс“. Для улучшения структурного качества буфером служила сглаживающая сверхрешетка InGaN/GaN. Активная область состояла из 5 квантовых ям/квантовых барьеров (КЯ/КБ) $\quad \operatorname{In}_{x} \mathrm{Ga}_{1-x} \mathrm{~N} / \mathrm{GaN}$ толщинами $3 / 12$ и $4 / 4$ нм с концентрацией индия 0.24 и 0.22 для образцов № CO1 и № П1 соответственно. Формировались слой $p$-AlGaN (толщина $20 \mathrm{HM}$ ), легированный магнием, с концентрацией акцепторов $N_{A}=2 \cdot 10^{19} \mathrm{~cm}^{-3}$ и слой $p$ - GaN (толщина 120 нм). Основные параметры исследуемых образцов представлены в табл. 1.

В табл. 1 приняты обозначения: $N$ - число КЯ, $S$ площадь поверхности кристалла.

Для каждого образца рассчитывалась ширина запрещенной зоны тройного соединения $\operatorname{In}_{x} \mathrm{Ga}_{1-x} \mathrm{~N} / \mathrm{GaN}$ с помощью следующего эмпирического выражения $[9,10]$ : $E_{g}=0.675 x+3.44(1-x)-1.3(1-x) x . \quad$ Подстановка значений концентрации индия $x=0.24, x=0.22$, установленных в процессе изготовления исследуемых образцов № CO1 и № П1, приводит соответственно к ширине запрещенной зоны сплава $\operatorname{In}_{x} \mathrm{Ga}_{1-x} \mathrm{~N} / \mathrm{GaN}$ $E_{g}=2.5$ и 2.6 эВ (табл. 1$)$.

В КЯ InGaN/GaN, учитывая ее узость, реализуется только один энергетический уровень $E_{1}$. Значения квантово-размерных энергетических уровней для всех 5 КЯ получены ранее авторами работы [8] в ходе интерпретации спектров электропропускания, снятых с образцов гетероструктур InGaN/AlGaN/GaN при разных обратных смещениях $p-n$-перехода (табл. 2). Из данных табл. 2 видно, что уровни испытывают смещение вдоль активного слоя $\mathrm{InGaN} / \mathrm{GaN}$. Это связано с возникающими на гетерограницах пьезоэлектрическими полями, ко- 
торые обусловлены наличием внутренних тангенциальных механических напряжений в слоях гексагональной сингонии материала $\mathrm{GaN}$.

Значения основных динамических параметров, таких как концентрация и подвижность 2ДЭГ, в активном слое $\mathrm{InGaN} / \mathrm{GaN}$ различаются для образцов № $\mathrm{CO} 1$ и № П1, что видно из рис. 2. Это можно объяснить особенностями удержания электронов в активном слое InGaN/GaN, влиянием гибридизации волновых функций, эффектом межслоевых деформаций и непараболичностью подзон.

В исследуемом диапазоне температур концентрация 2ДЭГ в гетероструктурах InGaN/AlGaN/GaN определялась методом измерения эффекта Холла. Регистрация температурных зависимостей холловской концентрации и подвижности 2ДЭГ проводилась на установке „HMS-3000“ в магнитных полях до 5 Тл в темноте в интервале от 2 до $300 \mathrm{~K}$ (рис. 2). Данные измерений показывают, что наблюдается немонотонная зависимость холловской концентрации 2ДЭГ в условиях исследуемого температурного режима.

Из рис. 2, $a$ видно, что в области низких температур $(40<T<200 \mathrm{~K})$ отмечается незначительное уменьшение холловской концентрации со средним постоянным значением $2.0 \cdot 10^{10}$ и $2.25 \cdot 10^{10} \mathrm{~cm}^{-2}$ для образцов № CO1 и № П1 соответственно. В области более высоких температур $T>200 \mathrm{~K}$ холловская концентрация 2ДЭГ резко возрастает и достигает в образцах № CO1 и № П1 значений $2.15 \cdot 10^{10}$ и $2.6 \cdot 10^{10} \mathrm{~cm}^{-2}$. Полученные в результате измерений функциональные зависимости можно объяснить следующим образом. Исследуемые гетероструктуры InGaN/AlGaN/GaN характеризуются широкой запрещенной зоной 2.6 эВ и большим значением концентрации донорной примеси $N_{D}=5 \cdot 10^{18} \mathrm{~cm}^{-3}$. Следовательно, в исследуемом диапазоне температур образцы № CO1 и № П1 обладают примесной проводимостью. Переход от примесной проводимости к собственной возможен при температуре $T_{s}=E_{g} / k_{\mathrm{B}} \ln \left(N_{c} N_{v} / N_{d}^{2}\right)$, где $E_{g}$ - ширина запрещенной зоны $N_{v}, N_{c}, N_{d}$ концентрация двумерных носителей заряда в валентной зоне, в зоне проводимости и на донорном уровне, $k_{\mathrm{B}}-$ постоянная Больцмана. Подстановка в формулу параметров гетероструктур InGaN/AlGaN/GaN приводит к температуре перехода $T_{s} \sim 10^{4} \mathrm{~K}$. Из этого следует, что для диапазона температур $90<T<170 \mathrm{~K}$ концентрация 2ДЭГ постоянна и определяется или примесной проводимостью, или носителями, возбужденными в 2ДЭГ фемтосекундными лазерными импульсами. Отсутствие в литературе данных, касающихся области истощения примеси в полупроводниках InGaN/AlGaN/GaN, не дает возможности утверждать, что концентрация 2ДЭГ для исследуемого температурного режима однозначно определяется примесной проводимостью. Данный вопрос требует дополнительных исследований.

Полученная температурная зависимость холловской подвижности 2ДЭГ типична для гетероструктур $\mathrm{InGaN} / \mathrm{AlGaN} / \mathrm{GaN}$ и одинаковым образом наблюдается для образцов № CO1 и № П1 (рис. 2,b). Подвижность
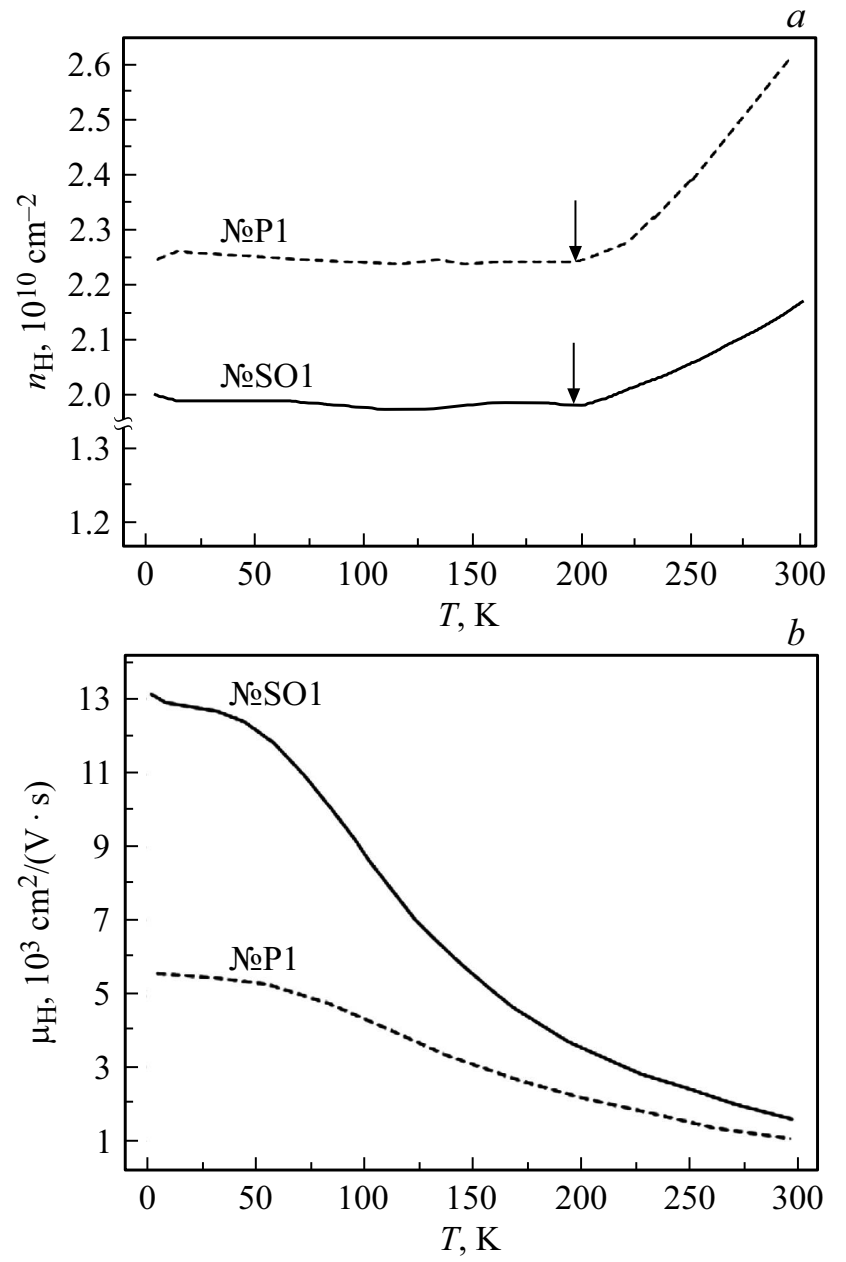

Рис. 2. Температурные зависимости холловской концентрации $(a)$ и холловской подвижности $(b)$ 2ДЭГ для образцов гетероструктур № CO1 и № П1. Стрелки указывают начало резкого возрастания функциональных зависимостей.

двумерных носителей уменьшается с ростом температуры, что объясняется усилением механизма рассеяния 2ДЭГ на оптических фононах при $T>85 \mathrm{~K}$.

Полоса пропускания ТГц-импульсов для образцов гетероструктур № CO1 и № П1 определяется толщиной образца и терагерцовым поглощением в эпитаксиальных слоях. Максимальное значение выходной мощности ТГцизлучения, наблюдаемое для образца № $\mathrm{CO} 1$, составило 8 мВт. Следовательно, можно говорить о соответствии исследуемых образцов гетероструктур области прозрачности в выбранном ТГц-диапазоне частот.

\section{3. Методика эксперимента. Обработка результатов}

Регистрация спектров эмиссии ТГц-излучения для образцов № CO1 и № П1 проводилась в геометрии на прохождение (рис. 3). Образцы возбуждались фемтосекундными лазерными импульсами с временны́ми окнами 


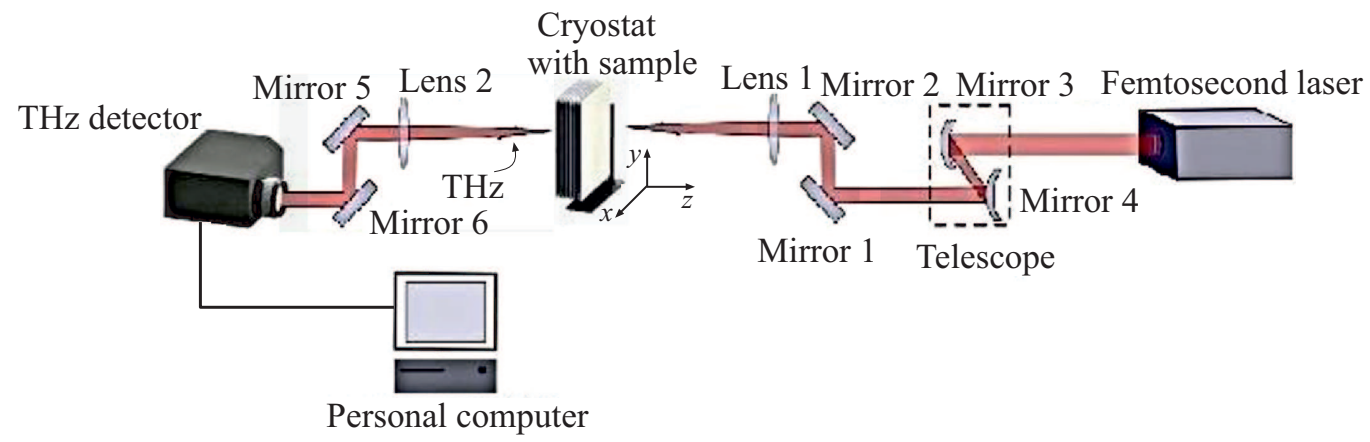

Рис. 3. Схема экспериментальной установки для регистрации ТГц плазмонных резонансов в образцах гетероструктур InGaN/AlGaN/GaN.

$t_{1}=130$ фс или $t_{2}=135$ фс на длине волны 797 нм в диапазоне температур от 90 до $170 \mathrm{~K}$ с шагом $30 \mathrm{~K}$. Частота следования импульсов фемтосекундного лазера составляла 60 МГц со средней выходной мощностью излучения 57 мВт. Лазерный пучок диаметром 1.8 мм проходил через оптическую систему при нормальном угле падения электрического поля импульсов Р-поляризации. Предварительно производилась юстировка оптического и ТГц путей излучения. Для выделения зондирующего импульса фемтосекундный лазерный пучок направлялся через оптическую систему задержки с настраиваемой длиной пути следования импульсов. Система задержки состояла из зеркал 1,2 , линзы 1 и телескопа, который содержал зеркало 1 и 2 с регулируемым между ними расстоянием. Выделение зондирующего импульса было проведено в целях стробирования детектора, чувствительного к электрическому полю ТГц-импульсов. Стробирование детектора ТГц-импульсов во временно́й области осуществлялось за счет изменения расстояния между зеркалами 3 и 4. На выходе из образца ТГцимпульсы, возбуждаемые в 2ДЭГ активной области $\mathrm{InGaN} / \mathrm{GaN}$, фокусировались линзой 2 и зеркалами 5,6. Настройка режима регистрации спектров эмиссии ТГцизлучения производилась путем регулирования длины следования ТГц-пучка импульсов между криостатом и детектором. Это расстояние подбиралось таким образом, чтобы быть сравнимым с толщиной исследуемых образцов гетероструктур InGaN/AlGaN/GaN. ТГцизлучение регистрировалось детектором со спектральным разрешением 0.5 ГГц и спектральным диапазоном 6 ТГц. Визуализация спектров эмиссии и их обработка производились с помощью персонального компьютера.

Исследовались опорный $E_{\mathrm{off}}(t)$ и модулированный $E_{\text {on }}(t)$ сигналы, пропускаемые через пустой криостат и криостат с образцом соответственно. На рис. 4, $a$ представлена временна́я зависимость сигнала детектора для $E_{\text {off }}(t)$ и $E_{\text {on }}$ образца № $\mathrm{CO} 1$, полученные при комнатной температуре.

Из рис. 4, $a$ видно, что основный пик модулированного сигнала, прошедшего через криостат с образцом, сдвинут по шкале времени на величину $\Delta t$ относительно
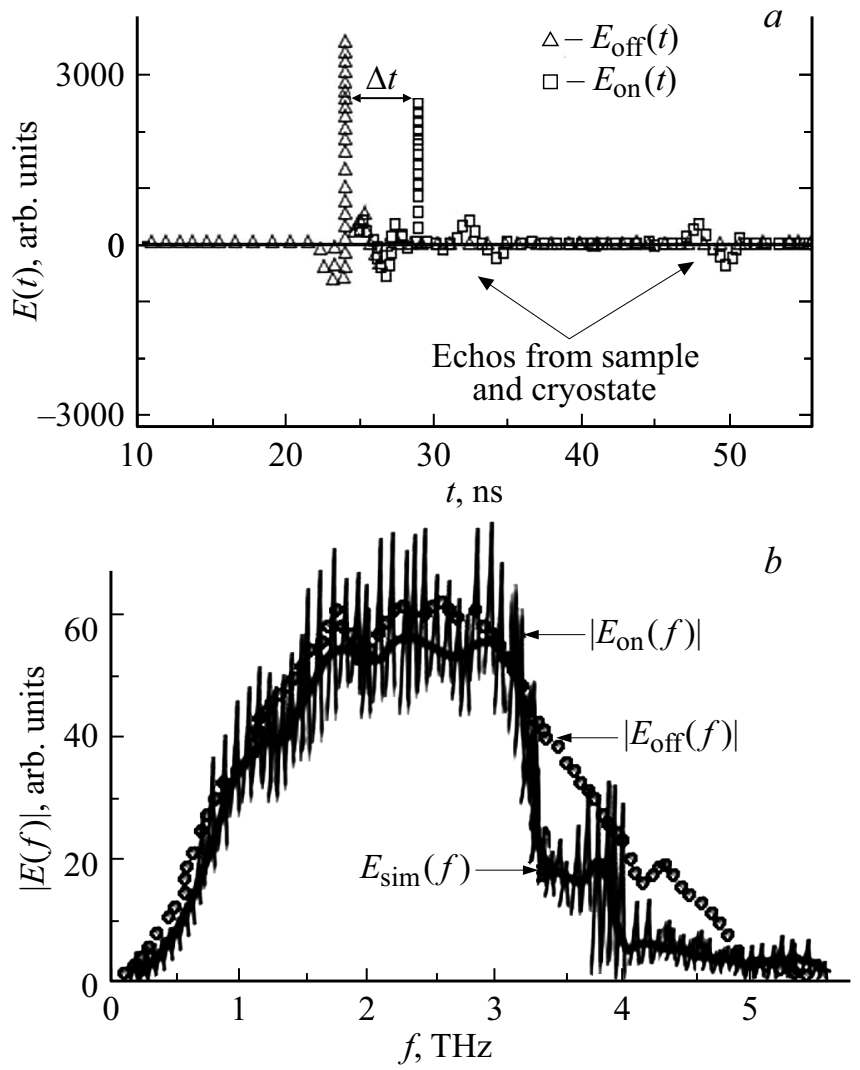

Рис. 4. Вид выходных данных детектора ТГц-импульсов. На рисунке показаны $(a)$ осциллограммы электрического поля сигнала ТГц-импульсов во временно́й области. Спектр получен для образца № CO1 при температуре $90 \mathrm{~K}$. На рисунке $(b)$ представлена соответствующая форма сигнала ТГц-импульсов, полученная методом быстрого фурье-преобразования (БФП)

опорного сигнала. Задержка регистрируемого детектором пучка ТГц импульсов обусловлена конечным временем перестройки системы 2ДЭГ, которое тем больше, чем ниже температура образца. Временем задержки ТГц-излучения в подложке можно было пренебречь, так как ее толщина составила 10 мкм. При моделировании динамики 2ДЭГ в гетероструктурах InGaN/AlGaN/GaN 
необходимо было учитывать существующие в КЯ механизмы рассеяния. Среди доминирующих в КЯ механизмов рассеяния выделяют рассеяние на шероховатостях гетерограницы InGaN/GaN, пьезоэлектрическое и кулоновское рассеяние на атомах примеси. Малые относительные отклонения дефектов гетерограниц $(2 \%)$, установленные методами дифрактометрии, и низкая (90 K) температура образцов № СО1, № П1 позволили не учитывать механизмы рассеяния в процессе моделирования. Следовательно, задержка импульса (рис. $4, a$ ) во временно́й области является характерным временем релаксации $\tau$ электронной системы в КЯ $\mathrm{InGaN} / \mathrm{GaN}$, которое определяет динамику 2ДЭГ в ответ на внешнее фемтосекундное излучение. Для образцов № CO1, № П1 время релаксации 2ДЭГ в КЯ $\mathrm{InGaN} / \mathrm{GaN}$ принимает соответственно значения $\tau=4.2$ и 3.8 нс.

В процессе исследования электрических свойств 2ДЭГ в КЯ $\mathrm{InGaN} / \mathrm{GaN}$ проведено моделирование оптических характеристик 2ДЭГ, в основе которого заложен метод быстрого преобразования Фурье (БПФ). Процедура БФП проведена с целью получения фурьекомпонент опорного и модулированного сигналов в частотной области:

$$
E_{\text {off,on }}(f)=\left|E_{\text {off,on }}(f)\right| \exp \left(i \varphi_{\text {off,on }}(f)\right),
$$

где $\left|E_{\text {off,on }}(f)\right|, \varphi_{\text {off,on }}(f)$ - фурье-амплитуды и фазы опорного и модулированного сигналов соответственно, $f$ - частота. В процессе сравнения отдельных частотнозависимых фурье-гармоник опорного и модулированного сигналов с помощью метода стробирования был выделен диапазон частот, в пределах которого 2ДЭГ демонстрирует резонансное поведение. Для характеристики спектров эмиссии ТГц-излучения, возбуждаемого системой 2ДЭГ в КЯ InGaN/GaN, были определены такие параметры, как спектральная мощность и фазовый сдвиг:

$$
\begin{gathered}
P_{i}(f)=\left|E_{\text {on }}(f)\right|^{2} /\left|E_{\text {off }}(f)\right|^{2}, \\
\Phi_{i}(f)=\varphi_{\text {on }}(f)-\varphi_{\text {off }}(f) .
\end{gathered}
$$

Для моделирования формы сигнала $E_{\text {on }}(f)$ проводилось разложение в ряд Фурье с вычисленными по формуле (1) фурье-компонентами опорного сигнала $E_{\text {off }}(f)$ и с комплексной функцией передачи $F(f)$ :

$$
E_{\mathrm{sim}}(t)=F(0) E_{\mathrm{off}}(0)+\sum_{k} F\left(f_{k}\right) E_{\mathrm{off}}\left(f_{k}\right) \exp \left(2 \pi i f_{k} t\right),
$$

где $F(f)$ была найдена путем решения уравнений Максвелла в гидродинамической модели высокочастотных свойств 2ДЭГ как отклик среды на плоскую волну с частотой $f, f_{k}=k / \Delta t, k=1,2 \ldots$ и $\Delta t-$ временно́е окно импульса. Функция $F(f)$ зависит от диэлектрического окружения 2ДЭГ в слоях гетероструктуры InGaN/AlGaN/GaN. В процессе нахождения функции $F(t)$ барьерный слой $\mathrm{GaN}$ и подложка моделировались как два разных слоя с комплексными диэлектрическими
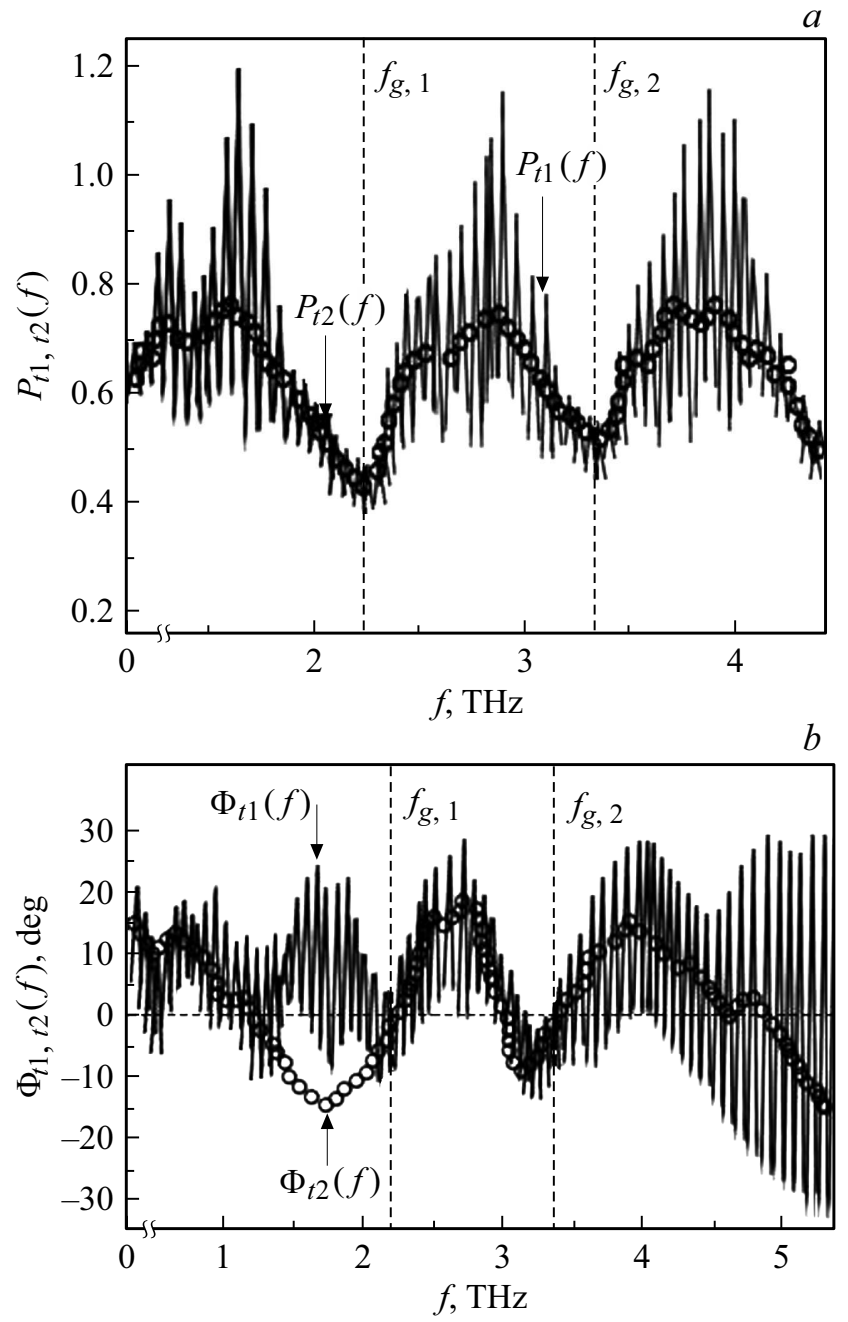

Рис. 5. Частотные зависимости $(a)$ мощности и $(b)$ фазы спектров эмиссии ТГц излучения. Спектры получены при возбуждении образца № CO1 фемтосекундными лазерными импульсами с временны́ми окнами $t_{1}=130$ и $t_{2}=135$ фс на длине волны $797 \mathrm{Hм}$.

проницаемостями $\varepsilon_{d}$ и $\varepsilon_{s}$. В выражениях для $\varepsilon_{d}$ и $\varepsilon_{s}$ учитывались компоненты $\operatorname{Im}\left[\varepsilon_{d}\right]$ и $\operatorname{Im}\left[\varepsilon_{s}\right]$, которые отвечают за потери энергии сигнала в плоскопараллельных слоях композитной гетероструктуры. Из рис. $4, b$ видно, что моделирование БПФ спектра ТГц-импульса хорошо согласуется с данными измерений.

БПФ сигнала $E_{\mathrm{sim}}(f)$ позволило произвести моделирование мощности $P_{t 1, t 2}(f)$ и фазовых сдвигов $\Phi_{t 1, t 2}(f)$ ТГц-сигнала с использованием различных временны́х окон $t_{1}, t_{2}$ лазерных импульсов. Результаты моделирования для образца № СО1 представлены на рис. 5.

На рис. 5 можно видеть модулированные полосами Фабри-Перо осцилляции функций $P_{t 1, t 2}(f)$ и $\Phi_{t 1 t 2}(f)$ в диапазоне частот от 1 до 5 ТГц. Эффект „бахромы“ на спектрах $P_{t 1, t 2}(f)$ и $\Phi_{t 1, t 2}(f)$ возникает вследствие отражения фемтосекундного лазерного импульса от подложки и его многократной интерфе- 
ренции в плоскопараллельных слоях гетероструктуры InGaN/AlGaN/GaN. Осцилляции Фабри-Перо наблюдаются для фемтосекундного импульса с временны́м окном $t_{1}=130$ фс и отсутствует у аналогичного лазерного импульса с временны́м окном $t_{2}=135$ фс. Подавление полос Фабри-Перо для второго лазерного импульса с $t_{2}=135$ фс было достигнуто путем вырезания дорожек сигнала перед первым „эхом“ на временно́й зависимости (рис. 4, $a$ ). Методом разворачивания фазы определялась полная фаза $\Phi_{t 1, t 2}(f)$, соответствующая числу периодов, укладывающихся в пределах разности хода длин волн падающего и прошедшего через криостат с образцом сигналов. Для этого к каждому значению фазы в диапазоне частот от 0 до 2.37 ТГц, соответствующих первому периоду плазмонных резонансов, прибавлялся множитель $2 \pi$. Заранее учитывалось, что скачки фазы отсутствуют в точках перехода через период. Значение разности хода длин волн также было установлено в ходе эксперимента. Результаты для лазерных импульсов с временны́ми окнами 130 и 135 фс показаны на рис. $5, b$.

На частотной зависимости мощности излучения рис. 5, $a$ наблюдаются провалы вблизи частот 2Д плазмонных резонансов, обозначенные как $f_{g 1}, f_{g 2}$. При температурах $>170 \mathrm{~K}$ особенности плазмонного резонанса в эксперименте не разрешались. Значения частот плазмонных резонансов, а также их количество являются характерной величиной низкоразмерной системы, определяющей электронные свойства 2ДЭГ присущие данной композитной структуре. Из рис. 5, $a$ видно, что значения первых двух резонансных частот образца № CO1 равны $f_{g 1}=2.37$ ТГц и $f_{g, 2}=3.48$ ТГц. В то же время на рис. 5, $b$ показано, что фазовый сдвиг вблизи плазмонных резонансов является точкой перегиба функции $\Phi_{t 1, t 2}(f)$, что соответствует минимумам в спектрах мощности $P_{t 1, t 2}(f)$. В ходе изучения частотных зависимостей, изображенных на рис. $5, b$, установлено, что фазовая модуляция превышает десятки градусов (до $14^{\circ}$ ). В данной работе это связывается с нелинейной динамикой электронной плазмы в плоскопараллельных слоях гетероструктуры InGaN/AlGaN/GaN.

Для рассматриваемых плазмонных структур резонансные частоты оценивались из закона дисперсии стробируемых 2Д плазмонов [11,12]:

$$
f_{g, n}=\frac{1}{2 \pi} \sqrt{\frac{4 \pi e^{2} N_{2 \mathrm{DEG}}\left|q_{n}\right|}{m^{*} \varepsilon_{0}\left(\varepsilon_{s}+\varepsilon_{d} \operatorname{coth}\left(\left|q_{n}\right| d_{2 \mathrm{DEG}}\right)\right)}},
$$

где $q_{n}=2 \pi n / P, n=1,2 \ldots \quad$ (в случае гетероструктур InGaN/AlGaN/GaN $n=2), P$ - период активного слоя $\mathrm{InGaN} / \mathrm{GaN}(P=15, P=8$ нм для образцов гетероструктур № CO1 и № П1 соответственно), $\varepsilon_{s}=11.6+i \alpha-$ диэлектрическая проницаемость подложки, $\varepsilon_{d}=10.5+i \beta-$ диэлектрическая проницаемость барьера [8], $m^{*}$ - эффективная масса, определяемая из (4), $\varepsilon_{0}$ - диэлектрическая постоянная,
Таблица 3. Параметры 2ДЭГ в КЯ $\mathrm{InGaN} / \mathrm{GaN}$ по данным частот двумерных плазмонных резонансов при температуре образцов $90 \mathrm{~K}$

\begin{tabular}{l|c|c|c|c|c|c}
\hline $\begin{array}{c}\text { Серия } \\
\text { образцов }\end{array}$ & $\begin{array}{c}N_{2 \mathrm{DEG}} \\
10^{10} \mathrm{~cm}^{-2}\end{array}$ & $\begin{array}{c}f_{g 1}, \\
\text { ТГц }\end{array}$ & $\begin{array}{c}g_{g, 2}, \\
\text { ТГц }\end{array}$ & $\begin{array}{c}\tau, \\
\mathrm{HC}\end{array}$ & $\left\langle m^{*}\right\rangle$ & $\begin{array}{c}\mu, 10^{3} \\
\mathrm{~cm}^{2} / \mathrm{B} \cdot \mathrm{c}\end{array}$ \\
\hline № CO1 & 2.0 & 2.37 & 3.48 & 4.2 & 0.42 & 1.7 \\
№ П1 & 2.25 & 2.22 & 3.56 & 3.8 & 0.40 & 1.5
\end{tabular}

Таблица 4. Зависимость эффективной массы 2ДЭГ в КЯ $\mathrm{InGaN} / \mathrm{GaN}$ от температуры

\begin{tabular}{l|c|c|c|c|c|c}
\hline \multicolumn{2}{c|}{ Серия образцов } & \multicolumn{5}{|c}{$T, \mathrm{~K}$} \\
\cline { 3 - 7 } \multicolumn{2}{l|}{} & 90 & 110 & 130 & 150 & 170 \\
\hline № CO1 & $m^{*}$ & 0.42 & 0.44 & 0.44 & 0.46 & 0.51 \\
№ П1 & $m^{*}$ & 0.40 & 0.42 & 0.42 & 0.45 & 0.48
\end{tabular}

$d_{2 \mathrm{DEG}}$ - глубина залегания 2ДЭГ относительно поверхности сапфировой подложки в гетероструктуре InGaN/AlGaN/GaN $\left(d_{2 \mathrm{DEG}}=28, d_{2 \mathrm{DEG}}=34\right.$ нм для образцов гетероструктур № CO1 и № П1 соответственно). В мнимых частях для комплексных диэлектрических проницаемостей $\varepsilon_{s}$ и $\varepsilon_{d}$ введены подгоночные параметры $\alpha=5.7, \beta=7.8$ которые учитывают радиационные потери в подложке и в области барьеров активного слоя InGaN/GaN. Значения подгоночных параметров подбирались в целях наилучшего соответствия смоделированного сигнала $E_{\mathrm{sim}}(t)$ с данными измерений рис. $4, b$ в процессе нахождения функции $F(f)$ (формула (3)). Для спектрального диапазона, лежащего ниже частоты первого плазмонного резонанса $f<f_{g, 1}$, учитывалось, что $\varepsilon_{s}$ и $\varepsilon_{d}$ являются бездисперсионными параметрами с постоянными значениями 11.6 и 10.5 соответственно [13]. Подстановка резонансных частот в формулу (4) дала возможность численно оценить эффективную массу $m^{*}$. В свою очередь с помощью найденного ранее (см. рис. 4, $a$ ) значения времени релаксации $\tau$ определя-

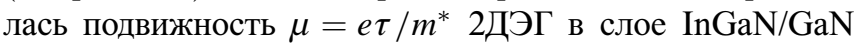
(табл. 3).

Спектры эмиссии для образцов гетероструктур № CO1 и № П1 были сняты в диапазоне температур от 90 до $170 \mathrm{~K}$ с шагом $30 \mathrm{~K}$. На основе смоделированного по формуле (3) сигнала были построены частотные зависимости мощности $P(f)$ и фазовых сдвигов при $\Phi(f)$ нагревании образцов от 90 до $170 \mathrm{~K}$, что показано на рис. 6.

На рис. 6 наблюдается красное смещение 2Д плазмонной резонансной частоты от значения, равного 3.48 ТГц (при $90 \mathrm{~K}$ ), до 2.96 ТГц (при $170 \mathrm{~K}$ ) для образца № CO1 и от значения 3.56 ТГц (при $90 \mathrm{~K}$ ) до 3.2 ТГц (при $170 \mathrm{~K})$ для образца № П1, которое идентифицируется как в спектрах $P(f)$, так и в спектрах $\Phi(f)$. В настоящей работе наблюдаемое красное смещение 2Д плазмонного 

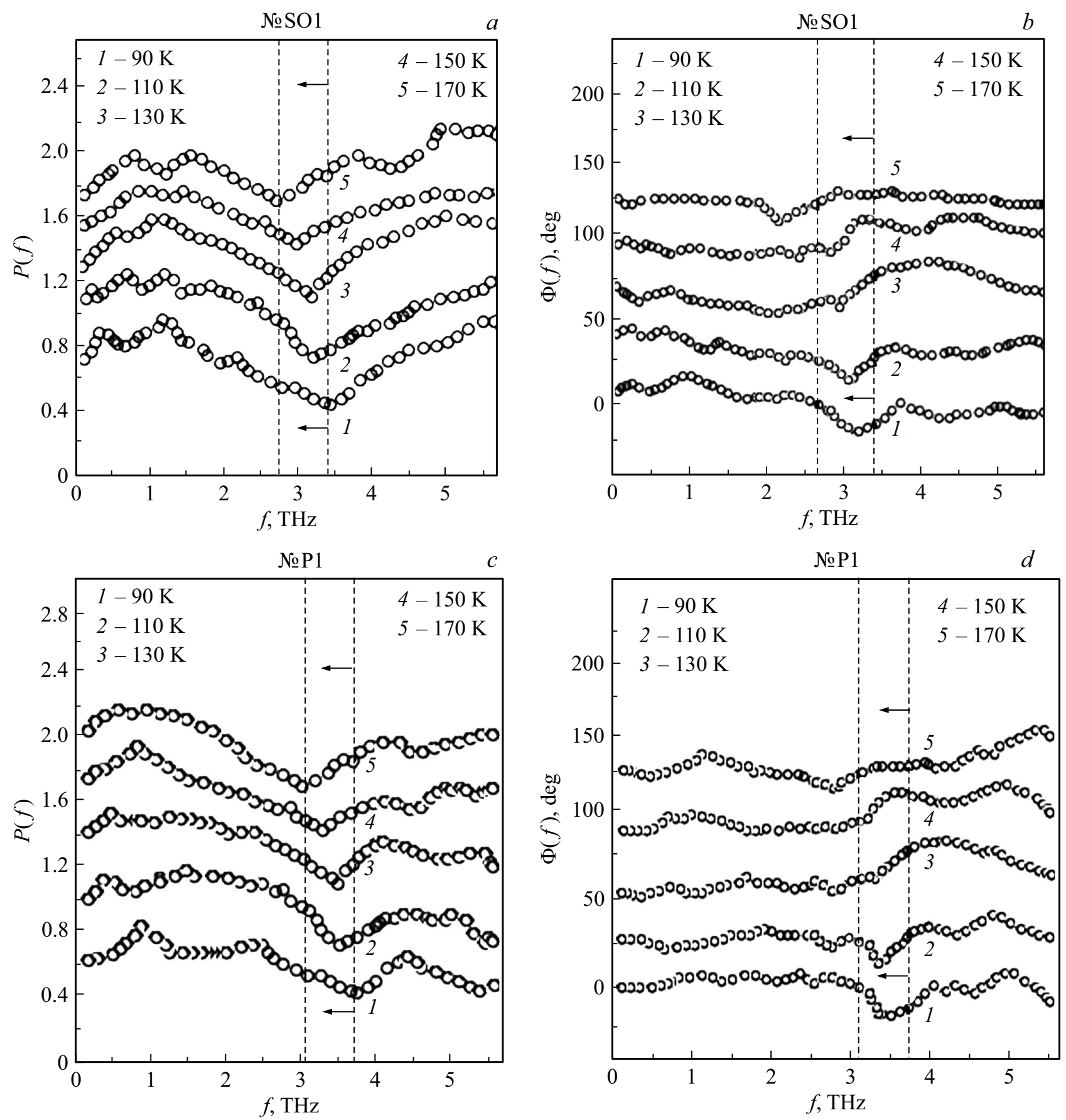

Рис. 6. На рисунках $a, b$ и $c, d$ представлены мощность и фаза спектров эмиссии ТГц излучения, полученные соответственно для образцов гетероструктур № CO1 и № П1 в диапазоне температур от 90 до $170 \mathrm{~K}$ с шагом 30 К. Пунктирные вертикальные линии обозначают положение частот плазмонных резонансов при температурах образца 90 и $170 \mathrm{~K}$. Стрелками обозначено красное смещение 2Д плазмонного резонанса.

резонанса связывается с температурной зависимостью эффективной массы $m^{*}(T)$ 2ДЭГ. В табл. 4 приведены рассчитанные на основании формулы (4) значения эффективной массы 2ДЭГ для серии образцов гетероструктур InGaN/AlGaN/GaN при температурах нагрева образца от 90 до $170 \mathrm{~K}$.

Из данных табл. 4 видно, что эффективная масса демонстрирует температурную зависимость с почти по- стоянными значениями для температур $<130 \mathrm{~K}$ и с быстрым увеличением при температурах $>130 \mathrm{~K}$. В настоящей работе наблюдаемая особенность связывается с перенормировкой эффективной массы в активном слое InGaN/GaN с ростом температуры.

Положение одного из 2Д плазмонных резонансов, например $f_{g, 2}$, в зависимости от температуры для образцов № CO1 и № П1 может быть изучено путем 
наблюдения за движением соответствующего минимума функции $P(f)$ или точки перегиба $\Phi(f)$, которые отмечены на рис. $6, a, b$ штриховыми линиями соответственно. Как видно из рис. 6 , увеличение температуры образца приводит к уширению резонансной линии и подавлению пиковой величины в спектре мощности (амплитуда изменилась с 0.27 при $90 \mathrm{~K}$ до 0.08 при $170 \mathrm{~K}$ для образца № CO1). В то же время наблюдается уменьшение фазовой модуляции рис. $6, b, d$ в фазовом спектре (амплитудный излом уменьшен с $20^{\circ}$ при $90 \mathrm{~K}$ до $6^{\circ}$ при $170 \mathrm{~K}$ для образца № CO1). Последнее можно объяснить снижением подвижности 2ДЭГ и времени релаксации в области высоких исследуемых температур.

\section{4. Заключение}

Таким образом, в настоящей работе на основании регистрации частот 2Д плазмонных резонансов предложен подход к определению времени релаксации, подвижности и эффективной массы 2ДЭГ, локализованного в КЯ $\mathrm{InGaN} / \mathrm{GaN}$. На основании обработки спектров эмиссии ТГц-излучения установлено, что 2ДЭГ в КЯ $\mathrm{InGaN} / \mathrm{GaN}$ характеризуется значением времени релаксации $4.2 \mathrm{Hc}$, подвижностью $1.7 \cdot 10^{3} \mathrm{~cm}^{2} / \mathrm{B} \cdot \mathrm{c}$ и эффективной массой $0.42 m$. Показано осциллирующее поведение мощности выходного ТГц-излучения с провалами в диапазоне частот от 1 ТГц до 5 ТГц, что связано с нелинейной динамикой 2ДЭГ в КЯ $\mathrm{InGaN} / \mathrm{GaN}$.

В процессе моделирования взаимодействия пучка фемтосекундных лазерных импульсов с плазмонной структурой исследована частотная и фазовая динамика стробируемых 2Д плазмонов. Для обработки и анализа ТГц-спектров эмиссии применялось быстрое фурьепреобразование, что позволило сравнить отдельные фурье-гармоники сигналов и определить амплитуду и фазу составляющих частот. На основании сравнения частотно-зависимых амплитуд и фаз ТГц-сигнала изучена динамика коллективных электронных возбуждений в 2ДЭГ. В процессе обработки ТГц-спектров при разных температурах нагрева образца обнаружен эффект перенормировки эффективной массы 2ДЭГ в активном слое InGaN/GaN, а также эффект модуляции фазы составляющих частот, что связано с нелинейной динамикой электронной плазмы в плоскопараллельных слоях гетероструктуры InGaN/AlGaN/GaN. Показано, что увеличение температуры приводит к красному смещению частот плазмонных резонансов с 3.48 до 2.96 ТГц (для образца № CO1) и от значения 3.56 до 3.2 ТГц (для образца № П1) для диапазона температур $90<T<170 \mathrm{~K}$. Поскольку резонансные частоты напрямую связаны со значением эффективной массой 2ДЭГ, удалось проследить и установить ее зависимость от температуры $m^{*}(T)$. В гетероструктурах $\mathrm{InGaN} / \mathrm{AlGaN} / \mathrm{GaN}$ наблюдается существенное $(20 \%)$ изменение эффективной массы в зависимости от температуры в диапазоне значений от
$0.42 m$ при $90 \mathrm{~K}$ до $0.51 m$ при $170 \mathrm{~K}$, что связано с перенормировкой $m^{*}$.

Показано, что на графиках частотной зависимости мощности ТГц-сигнала наблюдаются провалы вблизи плазмонных резонансов, что соответствует точке перегиба на функциональной зависимости фазового сдвига от частоты. Анализ спектров мощности и фазовых сдвигов излучаемых ТГц-импульсов позволил выявить значительную модуляцию фазы (до $14^{\circ}$ ) вблизи 2Д плазмонных резонансов, что может быть использовано в приложениях физики метаматериалов и терагерцовой цифровой голографии.

\section{Конфликт интересов}

Авторы заявляют, что у них нет конфликта интересов.

\section{Список литературы}

[1] А.В. Горбатова, Д.И. Хусяинов, А.Э. Ячменев, Р.А. Хабибуллин, Д.С. Пономарев, А.М. Буряков, Е.Д. Мишина. Письма ЖТФ, 46 (22), 10 (2020).

[2] В.И. Олешко, С.Г. Горина. Уч. записки физ. факультета Моск. ун-та, 5, № 155501, 1 (2015).

[3] Н.А. Гальчина, Л.М. Коган, Н.П. Сощин, С.С. Широков, А.Э. Юнович. ФТП, 41 (9), 758 (2007).

[4] В.А. Толмачев, Ю.А. Жарова, С.А. Грудинкин. Опт. и спектр., 90 (12), 1868 (2020).

[5] Р.Б. Любовский, С.И. Песоцкий, Г.В. Шилов, Е.И. Жиляева, А.М. Флакина, Р.Н. Любовская. Письма ЖЭТФ, 98 (3), 204 (2013).

[6] Xi-Cheng Xhang, Jingzhou Xu. Introduction to $\mathrm{THz}$ Wave Photonics (Springer US, 2010).

[7] B. Richard, M. Schasfoort. Handbook of Surface Plasmon Resonance (Royal Society of Chemistry, 2017).

[8] А.Э. Асланян, Л.П. Авакянц, А.В. Червяков, А.Н. Туркин, С.С. Мирзаи, В.А. Курешов, Д.Р. Сабитов, А.А. Мармалюк. ФТП, 54 (4), 420 (2020).

[9] P. Schley, R. Goldhahn, G. Gobsch, M. Feneberg, K. Thonke, X. Wang, A. Yoshikawa. Phys. Status Solidi B, 246 (6), 1177 (2009).

[10] P.R. Pelá, C. Caetano, M. Marques, L.G. Ferreira, J. Furthmuller, L.K. Telles. Appl. Phys. Lett., 98 (15), 151907 (2011).

[11] A.V. Chaplik. Surf. Sci. Rep., 5, 289 (1985).

[12] S.J. Allen, D.C. Tsui, R.A. Logan. Phys. Rev. Lett., 38, 980 (1977).

[13] С.Ю. Давыдов. ФТТ, 51 (6), 1161 (2009).

Редактор Г.А. Оганесян 


\section{Investigation of the parameters \\ of a two-dimensional electron gas \\ in quantum wells InGaN/GaN by the method of terahertz plasmon resonance}

\section{E.R. Burmistrov, L.P. Avakyants}

Lomonosov Moscow State University

(Faculty of Physics),

119991 Moscow, Russia

Abstract A new approach to determining the parameters of a two-dimensional electron gas (2DEG) in $\mathrm{InGaN} / \mathrm{GaN}$ quantum wells is proposed. It is based on the method of terahertz spectroscopy with time resolution, within the framework of which the terahertz frequencies of two-dimensional plasmon resonances excited in the studied samples of InGaN/AlGaN/GaN heterostructures by femtosecond laser pulses at a wavelength of $797 \mathrm{~nm}$ were recorded. Oscillating behavior of the output terahertz radiation power with minima in the frequency range $1-5 \mathrm{THz}$ is shown, which is associated with the excitation of plasmon oscillations in a two-dimensional electron gas localized in an $\mathrm{InGaN} / \mathrm{GaN}$ quantum well. During the processing of terahertz spectra, the effect of renormalization of the effective mass of two-dimensional electron gas, as well as phase modulation near the frequencies of plasmon resonances with an increase in the temperature of the sample from 90 to $170 \mathrm{~K}$, was found. The proposed method is non-contact and can be used in a wide temperature range. 